DOI: https://doi.org/10.46630/phm.12.2020.26

Stefan J. Zdravković ${ }^{1}$

Article de recherche

Université de Niš

УДК 371.3::811.133.1]:004.738.5

Faculté de philosophie ${ }^{2}$

Reçu : le 21/2/2020

Département de langue et de littérature françaises

\title{
LE BLOG PÉDAGOGIQUE COMME OUTIL D'ENSEIGNEMENT/ APPRENTISSAGE DU FLE
}

Dans la présente analyse nous tenterons de mettre en lumière le blog pédagogique avec tous ses avantages et inconvénients, qui, dans la nouvelle ère technologique, émerge en tant qu'un outil d'enseignement/apprentissage à part entier. En partant de la définition de Matagne, Berhin et Orban de Xivry (2007) qui par un blog pédagogique entendent un blog défini par « une pratique et/ou une diffusion de contenu aidant au processus d'apprentissage ou résultant de celui-ci, dans le monde de l'enseignement » l'objectif principal de cette étude est de confirmer ou d'infirmer l'hypothèse que le blog pédagogique apporte de nouvelles tendances dans la préparation des cours du FLE, qu'il est un facteur de motivation des apprenants et qu'il fait naître de meilleurs liens sociaux entre le concepteur du blog/l'enseignant et le public/les apprenants. Nous vérifierons si cette interaction entre l'enseignant et les apprenants, qui se produit dans ce nouvel espace numérique enrichi par les contenus multimédias, crée de nouveaux potentiels éducatifs et un certain prolongement de la classe. Cette étude ne donnera pas qu'un aperçu théorique de ce nouvel outil d'enseignement, mais aussi elle proposera une analyse qualitative des blogs pédagogiques d'après l'enquête menée auprès de nos étudiants, qui ont travaillé avec le blog en tant qu'apprenants et en tant que concepteurs de leurs propres blogs pédagogiques.

Mots-clés : blog, TICE, FLE, enseignant, apprenant

\section{Introduction}

Dans la présente communication, à part la détermination théorique du blog pédagogique, qui est un outil d'enseignement tout nouveau, nous

\footnotetext{
${ }^{1}$ stefan.zdravkovic@filfak.ni.ac.rs

${ }^{2}$ Cet article est soutenu par le Ministère de l'éducation, de la recherche et du développement technologique de la République de Serbie. Cet article est rédigé dans le cadre du projet scientifique Les langues, les littératures et les cultures romanes et slaves en contact et en divergence (no 81/1-17-8-01) soutenu par la Faculté de philosophie de l'Université de Niš, l'AUF (Agence universitaire de la Francophonie) et l'Ambassade de France en Serbie.
} 
ferons une analyse des avantages et des inconvénients de l'utilisation du blog pédagogique dans l'enseignement/apprentissage du FLE. Notre hypothèse initiale est que le blog pédagogique apporte de nouvelles tendances dans la préparation des cours du FLE, qu'il est un facteur de motivation des apprenants et qu'il fait naître de meilleurs liens sociaux entre le concepteur $\mathrm{du}$ blog/l'enseignant et le public/les apprenants. Nous confirmerons ou infirmerons cette hypothèse initiale en analysant les réponses obtenues de la part des participants à l'enquête menée. Les répondants à l'enquête étaient nos étudiants qui ont travaillé avec le blog en tant qu'apprenants de TICE et en tant que futurs enseignants/concepteurs de leurs propres blogs pédagogiques dédiés à l'apprentissage du FLE.

\section{Les TICE et la réalité numérique}

Avant de parler de l'utilisation des blogs dans l'enseignement, il faut mentionner le terme TICE. C'est l'acronyme qui signifie Technologies de l'Information et de la Communication pour l'Éducation. D'après le Dictionnaire pratique de didactique du FLE les enjeux des TICE sont considérables puisqu'elles " donnent aux apprenants l'accès au savoir » tout en mettant « les richesses du multimédia au service de la modernisation pédagogique ». De même, les TICE « donnent aux futurs citoyens la maitrise des nouveaux outils de communication » et mettent en lumière la compétence interculturelle (ROBERT 2008 : 199).

L'autre terme synonyme utilisé en didactique est la technologie éducative qui est défini d'après Pedagoški leksikon [Lexicon de pédagogie] en tant que " processus systématique et organisé d'application de techniques et technologies contemporaines dans le but d'améliorer la qualité du processus éducatif (efficacité, optimalité, faisabilité, etc.). C'est une façon systématique de conception et d'évaluation du processus éducatif, c'est-à-dire de l'apprentissage et de l'enseignement, en utilisant la technique d'enseignement contemporaine »(PEDAGOŠKI LEKSIKON 1996 : 334). La technologie éducative se développe grâce à l'accès presque illimité à Internet et à des ordinateurs portables, des tablettes et des smartphones (qui, d'ailleurs, a beaucoup changé notre vie au XXI siècle). De cette façon l'enseignement du français langue étrangère a beaucoup évolué de l'enseignement du dernier siècle, qui n'a pas eu l'accès (instantané) à tous ces médias. En adaptant et en créant de nouveax dispositifs numériques à exploiter pour la classe de langue l'enseignant du $\mathrm{XXI}^{\mathrm{e}}$ siècle devient " producteur et non seulement consommateur de contenus numériques » (CORDINA, RAMBERT et al. 2017 :12). Cela sous-entend un certain niveau de littératie numérique qui comprend 
« une vaste capacité de participer à une société qui utilise la technologie des communications numériques dans les milieux de travail, au gouvernement, en éducation, dans les domaines culturels, dans les espaces civiques, dans les foyers et dans les loisirs » (HOECHSMANN, DEWAARD $2015: 5$ ).

Si l'enseignant dispose d'une connexion Internet et d'un ordinateur qui peut être projeté sur un écran dans la classe « la quantité de documents authentiques disponibles à être vus, écoutés, et lus par les apprenants devient infinie (chansons, articles de presse, vidéos, films, émissions radio, images) » (CORDINA, RAMBERT et al. 2017 : 14). L'introduction du numérique dans l'enseignement/apprentissage du FLE qui se déroule du début de ce siècle, n'est pas une révolution pédagogique, mais plutôt une innovation pédagogique enrichissant des courants pédagogiques déjà existants (Ibid.: 16). Selon l'auteur Lebrun « les bénéfices les plus flagrants de l'intégration des technologies de l'information et de la communication dans l'enseignement sont à rechercher parmi les méthodes ouvertes et actives [parmi lesquelles le blog] car elles contribuent à la préparation des personnalités "fortes" que la société revendique » (LEBRUN 2002 : 186).

\subsection{Le terme $b \log$}

D'après l'Encyclopédie Universalis le terme blog provient du motvalise weblog, qui est une contraction des mots anglais web et $\log$ signifiant « carnet de bord sur Internet ». Le blog a vu le jour en 1999 aux États-Unis. Dans le dispositif du blog les deux services précédemment distincts ont été réunis pour la première fois : l'un comprend les outils d'autopublication (les pages ou les sites personnels), l'autre les outils de communication collective (les forums, les chats) (ENCYCLOPÆDIA UNIVERSALIS [en ligne], consulté le 5 août 2019). Les blogs ont apparu en tant qu'une sorte de « filtres de l'Internet », c'est-à-dire que les concepteurs de blogs y ont commencé à présenter les informations les plus pertinentes suivies par des commentaires personnels. Au début ce n'étaient que les designers web et les développeurs de logiciels qui écrivaient les blogs (TRÉDAN 2005 : 2). Dans les années 2000 le blog a connu un essor très important et le nombre de blogs a rapidement atteint plusieurs millions. Quant aux types de blogs, il y a ceux sur lesquels les internautes publient de différentes sources multimédias et ceux qui sont spécialisés d'après le type du contenu publié : vlogs (vidéoblogs), photoblogs ou audioblogs (ENCYCLOPÆDIA UNIVERSALIS [en ligne], consulté le 5 août 2019).

Le Petit Robert définit le blog comme un « carnet de bord sur internet, animé par un individu ou une communauté » (LE PETIT ROBERT 2006). Le Meur et Beauvais ont défini le blog comme un « site Web personnel composé essentiellement de billets d'actualité, publiés jour après jour ou au gré des 
humeurs et apparaissant selon un ordre antéchronologique, les plus récents figurant en haut de pages » (LE MEUR, BEAUVAIS 2005 : 1) Les articles publiés sur un blog peuvent être commentés par les internautes, et on peut y ajouter des liens externes menant vers d'autres sites traitant une thématique similaire. L'auteure Roboredo Seara trouve que « la facilité de la création en ligne et l'interactivité sont[...] deux facteurs essentiels du développement vertigineux du phénomène blogging » (ROBOREDO SEARA $2010: 242$ ).

Il existe toute une gamme de termes synonymes du mot blog: blogue, weblog, carnet web, cybercarnet, joueb, journal web, bloc-notes etc. (KLEIN (dir.) 2007 : 11). Pourtant, le terme anglais blog (ensemble avec le mot anglais weblog dont il est la forme contractée) reste le plus répandu dans l'espace francophone et c'est pour cette raison que nous avons décidé de l'utiliser dans notre article.

\subsection{La visibilité sur Internet}

Quant aux résultats des recherches ou de bonnes pratiques de l'enseignement du FLE, le blogging peut nous aider à « accroître sa visibilité », à repenser nos propres pratiques, à échanger les idées (HÉNAFF 2009 : 386). Cette visibilité accrue grâce au blog comprend les contenus pédagogiques publiés. Par le biais des blogs les enseignants partagent des contenus pédagogiques prêts à l'utilisation, ainsi que des contenus mulitmédias qui peuvent être didactisés et exploités en classe du FLE. De plus, tous les contenus une fois publiés restent à la disposition des enseignants, des apprenants et du monde entier (CORDINA, RAMBERT et al. 2017 : 18).

\subsection{Blogs pédagogiques et leurs types}

Matagne, Berhin et Orban de Xivry définissent le blog pédagogique comme " une pratique et/ou une diffusion de contenu aidant au processus d'apprentissage ou résultant de celui-ci, dans le monde de l'enseignement » (KLEIN (dir.) 2007 : 126). Selon Aaron Patric Campbell (2003) il existe trois types de blogs pédagogiques : le blog du tuteur (" the tutor blog »), le blog de l'apprenant («the learner blog ») et le blog de la classe (" the class blog ») (CAMPBELL : 2003).

Le blog du tuteur représente un espace de cours prolongé : l'enseignant peut y publier les leçons, les textes intéressants, les activités et les devoirs pour ses apprenants. Il peut y ajouter toutes les précisions qui concerneront par exemple la date limite pour rendre les devoirs à la maison etc. C'était le cas aussi avec le blog que nous avons utilisé dans notre pratique de l'enseignement d'où ce témoignage d'un étudiant qui parle des plus grands avantages du blog utilisé par le professeur : « ce sont les consignes [des activités] étant toujours 
disponibles pour consulter et c'est pratique parce qu'il y avait toujours un lien sur Internet où se trouvent les devoirs ». Grâce aux liens externes qui peuvent être publiés sur un blog menant vers les sites utiles pour l'apprentissage de la langue française les apprenants peuvent approfondir leurs savoirs et savoirfaire en lisant les sites qui existent déjà (avec de différents types de sources telles que audio, vidéo, textes, images, quiz interactifs etc.). Cette liste de liens externes offre une possibilité d'apprentissage en autonomie avec un certain suivi de la part de l'enseignant. De cette façon les apprenants feraient des recherches des sites en langue cible et seraient plus à l'aise avec ce type de documents authentiques. Il est souhaitable que le blog ait une présentation du cursus et un schéma d'évaluation. Ceci est indispensable surtout pour l'enseignement à distance (Ibid.).

Le blog de l'apprenant peut être fait par un apprenant ou par un groupe d'apprenants. Les blogs créés par des apprenants sont utiles sur plusieurs aspects. Ils peuvent développer non seulement les compétences langagières chez les apprenants, mais aussi ils rendent les apprenants créatifs et les aident à acquérir les savoirs interdisciplinaires et les compétences interculturelles. Quant aux blogs créés par plusieurs apprenants, cela favorise la collaboration entre les membres du groupe et ainsi naît l'esprit du groupe et la satisfaction d'avoir créé quelque chose en commun. Enfin, en écrivant le blog l'apprenant devient plus expérimenté avec les moteurs de recherche pour les documents authentiques en français (Ibid.).

Quant au blog de la classe Campbell y voit soit un carnet en ligne où les apprenants d'une classe sont invités à publier des messages, des images, des liens etc. et qui concernent le thème abordé en cours, soit une sorte de développement d'un projet dédié à l'apprentissage de la langue étrangère ou encore un échange virtuel international (Ibid.).

\subsection{Les avantages et les inconvénients du blog pédagogique}

Un grand avantage du blog est sa facilité de création et de mise à jour régulière ou à son propre gré. De cette façon, tout le monde est, en quelque sorte, invité à créer, publier, échanger, commenter en enrichissant ses propres savoirs. L'essor du blog va de pair avec une forte croissance de l'utilisation d'Internet et sa vulgarisation (DESAVOYE, DUCAMP et al. : 2005). Aujourd'hui n'importe qui peut créer son propre espace sur Web et y diffuser du contenu, ce qui était, pendant des années, exclusivement le travail des programmeurs. D'après Hoechsmann et Dewaard les blogs « sont idéalement conçus pour le dialogue et la création d'une communauté en classe. Commenter le blog d'une autre personne peut contribuer à une conversation numérique sur des enjeux ou des questions présentées d'une variété de perspectives » (HOECHSMANN, DEWAARD 2015 : 37). 
La création d'un blog ne demande ni sources financières ni savoirs supplémentaires que possède un programmeur, vu qu'aujourd'hui plusieurs plateformes offrent la création gratuite des blogs (blogger, wordpress etc.) et que cette création se fait en quelques clics de souris. De plus, la mise à jour des informations et des articles du blog est très facile et rapide. Une fois le blog créé, tout le monde participant au processus de la formation est invité à publier et/ou commenter. La réactivité des apprenants est très importante parce que de cette façon l'enseignant peut se rendre compte de la créativité de ses apprenants, de ce qu'ils pensent et il peut éventuellement les évaluer. L'enseignant-blogueur peut aussi recevoir des commentaires d'autres enseignants appartenant à son champ disciplinaire. De cette façon les enseignants ont la possibilité d'échanger des idées et l'enseignant-blogueur peut obtenir une sorte de feedback quant à son journal éducatif. Grâce aux TICE l'enseignant peut instantanément remédier à un problème qui se pose devant ses apprenants et qui semble difficile à résoudre. Force est de constater qu'une sorte de prolongement de la classe naît avec l'apparition du blog : sur blog le cours ne finit pas après l'école. Enfin, en utilisant le blog, les apprenants n'améliorent pas que leurs compétences linguistiques et interculturelles, mais aussi leurs compétences pragmatiques informatiques.

Avec l'intégration du numérique dans l'enseignement (le blog pédagogique y compris) la notion du « maître » disparaît progressivement et naît le guide-accompagnateur de ressources numériques (CORDINA, RAMBERT et al. 2017 : 19). De là naissent de meilleurs liens sociaux entre l'enseignant et les apprenants, ce qui est surtout encourageant pour les plus timides. Tous « les commentaires, retours et critiques » publiés sur les blogs des apprenants encouragent les apprenants à s'exprimer, «à défendre leurs idées, leurs écrits face au public » (HÉNAFF 2009 : 386). Même les apprenants plus timides sont invités à s'exprimer soit à l'écrit, soit à l'oral (en utilisant les outils pour l'enregistrement de la voix, par exemple). Dans les blogs s'exprimer devant le public prend un autre sens, et l'apprenant n'est plus gêné par le public dans une salle de classe. Les tâches proposées sur les blogs peuvent être très motivantes si elles sont bien précises et si les apprenants en connaissent les objectifs.

Il est incontestable que les blogs ont de multiples atouts, mais il se pose la question : Est-ce que les blogs ont certains inconvénients ? Il est vrai que blog aide les apprenants dans le processus de l'acquisition des savoirs et des savoir-faire. Pourtant, il ne peut pas remplacer le contact personnel entre l'enseignant et ses apprenants. Ceci est indispensable pour l'interaction orale entre l'enseignant et ses apprenants et entre les apprenants eux-mêmes. L'autre inconvénient, qui est valable surtout pour l'auteur du blog, est que le blog demande des heures de travail supplémentaire pour la publication des articles. 
Mais si les heures consacrées à l'organisation et à la présentation visuelle du blog portent leurs fruits, alors le temps n'est pas gaspillé. Plus précisément, si le blog aide au processus d'acquisition des compétences linguistiques, interculturelles et pragmatiques et s'il motive les apprenants à travailler, cela représente une grande récompense pour l'enseignant. Enfin, certains prérequis de base sont indispensables : un certain niveau de littératie numérique de base est nécessaire pour organiser le blog et pour commencer à publier les articles. Une contrainte technique qui se pose devant les enseignants, les apprenants et les écoles est l'accès à Internet.

\subsection{Intégration des blogs dans des dispositifs scolaires}

D'après les auteurs Lapointe et Drouin le blog intégré dans les dispositifs scolaires de l'enseignement possède trois rôles fondamentaux. Tout d'abord le blog représente un espace d'écriture : les enseignants amènent les apprenants à écrire, à commenter et à dialoguer en pratiquant leur français. Puis, le blog sert d'instrument de gestion de contenu, c'est-à-dire sur le blog on peut afficher « les exercices pratiques, les références bibliographiques, les informations complémentaires au cours » (en évitant les vieilles photocopies et en sauvant du papier). Le troisième rôle fondamental des blogs est le fait qu'ils sont devenus un lieu de discussion vu que "si on attire les étudiants en un lieu unique, il devient plus facile de les inciter à intervenir, commenter, dialoguer, entre eux ou avec les autres » (LAPOINTE, DROUIN 2007 : 135-136).

Ce qui rend l'intégration des blogs dans des dispositifs scolaires difficile, ce sont les contraintes techniques : c'est-à-dire si l'institution scolaire et les apprenants possèdent des ordinateurs (avec ou sans projecteur), des tablettes ou des smartphones et s'ils ont l'accès à Internet. L'autre contrainte est un minimum de compétence informatique et maitrise technique du blog de la part des enseignants.

\section{Blog pédagogique dans le contexte universitaire de notre pratique de l'enseignement}

Dans notre pratique de l'enseignement des Technologies de l'Information et de la Communication pour l'Éducation (TICE) au Département de la langue et littérature françaises de la Faculté de Philosophie (Université de Niš), nous avons mené les blogs pour y publier toutes les activités qu'il fallait faire pendant le semestre. ${ }^{3}$ Les articles publiés sur les blogs comportaient aussi des explications sur la manière de réaliser certaines activités ou d'utiliser

${ }^{3}$ Nos blogs sont disponibles aux adresses suivantes : http://tice2019.blogspot.com/ et https:// ticequatriemeannee.blogspot.com/. 
certaines plateformes afin de créer des activités pour un cours du FLE. Toutes les explications étaient enrichies de photos, vidéos, textes ou liens menant vers d'autres sites. De cette façon, le blog a remplacé les photocopies, et il était disponible en tout lieu et à chaque instant sur les ordinateurs ou les smartphones des étudiants. De plus, ce dispositif a fait en sorte que nous puissions optimiser le temps dans la classe, vu que certains prolongements du cours en ligne ont eu lieu avant et après les cours en présentiel. Dans le cadre de la matière que nous avons enseignée nos étudiants, futurs enseignants du FLE, ont eu également la possibilité de faire leurs propres blogs dédiés à l'apprentissage du FLE de différents niveaux (de A1 à B2). ${ }^{4}$

Afin de mieux comprendre et analyser l'expérience de nos étudiants dans l'utilisation du blog pédagogique nous avons mené une enquête auprès des étudiants concernés. Au total il y avait 24 répondants. ${ }^{5}$ Toutes les réponses étaient anonymes. Il y avait au total 12 questions : 4 questions fermées et 8 questions ouvertes. D'après les réponses aux questions fermées nous avons fait une analyse quantitative (du blog pédagogique en tant que facteur de motivation et de l'utilité du blog pédagogique) et les réponses aux questions fermées nous étaient très utiles à rédiger l'analyse qualitative (des côtés positifs et des côtés négatifs du blog pédagogique). L'enquête a eu pour objectif d'identifier les avantages et les inconvénients des blogs pédagogiques pour l'enseignement/apprentissage du FLE. Les étudiants ont été demandés d'expliquer les caractéristiques positives et négatives des blogs pédagogiques des deux côtés : du côté de l'apprenant des TICE et du côté du concepteur de son propre blog dédié à l'enseignement/apprentissage du FLE. Avant de mener cette enquête on s'est posé deux questions principales : Le blog est-il motivant pour les étudiants? Est-ce que le blog est utile à l'apprentissage ?

\subsection{Blog pédagogique en tant que facteur de motivation}

La majorité des participants à l'enquête trouvent que le blog a été motivant pour leur apprentissage. Presque les deux tiers (58,33\%) des étudiants pensent que le blog a été très motivant pour eux, tandis qu'un tiers $(33,33 \%)$ trouve le blog motivant. Seulement $8,33 \%$ des étudiants trouvent le blog peu motivant, et personne ne trouve le blog pas si motivant ou pas motivant (voir le graphique $n^{\circ} 1$ ).

\footnotetext{
${ }^{4}$ Voici deux exemples de blogs de mes étudiants : https://ticecafe1.blogspot.com/, https:// lafrancophonietice2019.blogspot.com/. Je tiens à remercier mes étudiantes Monika Tanić, Katarina Radenković, Milica Stevanović et Sofija Filipović pour leur permission de citer leurs blogs en tant qu'exemples dans cet article.

${ }^{5}$ Je remercie chaleureusement mes étudiants d'avoir participé à cette enquête.
} 
Stefan J. Zdravković

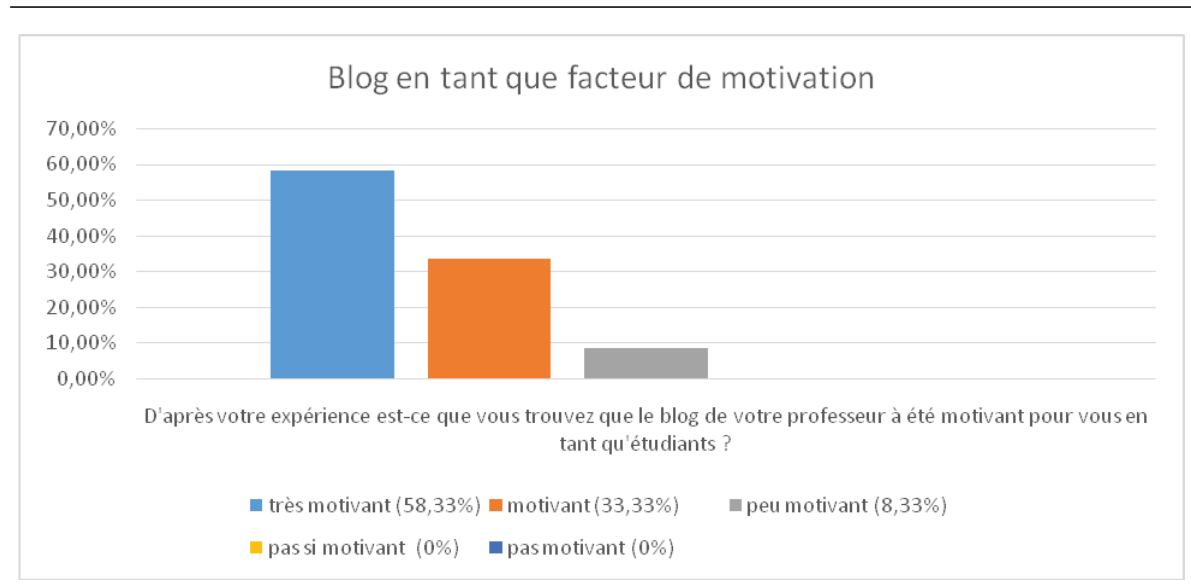

\section{Le graphique $n^{\circ} 1$}

Ces réponses favorables sont suivies par les commentaires souvent très positifs. La source de cette motivation se trouve tout d'abord dans le fait que c'est une nouvelle approche peu exploitée dans leur formation : "c était une nouveauté dans mon éducation, et j'étais motivé d'explorer ce moyen d'enseignement »; ou dans le fait que tout simplement " c'est motivant et intéressant d'utiliser la nouvelle technologie pour les cours ». Une étudiante trouve que le blog l'a rendue plus créative pour créer ses propres exercices et son propre blog. Un participant à l'enquête croit qu'il était motivé parce que le blog utilisé en cours "était bien écrit, avec une communication directe, avec des petits messages motivants ». Les apprenants ont aimé le blog parce que «c'était une approche plus ludique » et ils constatent qu'aussi le choix des exercices et des jeux avait un effet important sur leur motivation. D'après un participant à l'enquête, lors de l'utilisation du blog en cours ou en dehors de la salle de classe il apprenait sans s'en apercevoir, ce qui veut bien dire qu'il était motivé. Pour créer un blog pédagogique motivant il est important non seulement de faire de bonnes activités avec des objectifs bien précis, mais aussi de bien présenter le contenu : «premièrement, la présentation et l'organisation visuelles du blog sont très motivantes pour les apprenants. Il y a beaucoup d'activités intéressantes avec les consignes claires qui motivent les apprenants à travailler $»$.

\subsection{Le blog vu de la part des apprenants}

Comme nous avons déjà dit, l'enquête menée auprès des 24 étudiants des TICE comportait deux parties principales : l'une concernant le point de vue de l'apprenant des TICE, qui suivait le blog de l'enseignant, l'autre concernant le point de vue du jeune concepteur du blog (futur enseignant) dédié à l'apprentissage du FLE. 
D'après leur expérience de l'apprenant des TICE, les participants à l'enquête ont trouvé certains inconvénients à l'utilisation du blog. En premier lieu, et deux apprenants l'ont souligné, c'est qu'afin d'utiliser le blog il est indispensable de posséder un minimum de littératie numérique, c'est-à-dire «il faut " apprendre » à utiliser le blog » et c'est la raison pour laquelle «c'est seulement un peu difficile au début de comprendre comment ça fonctionne». L'autre inconvénient du blog utilisé en cours était le fait que "parfois on ne peut pas trouver vite ce que l'on cherche ", mais cela dépend toujours de l'organisation d'un blog bien précis, et cela n'est pas le cas avec tous les blogs. De toute façon, on tire la conclusion que la bonne organisation du blog est indispensable à la visibilité de tous ses éléments. Et, enfin, ce qu'on trouve parmi les commentaires concernant les points négatifs du blog est une contrainte technique indispensable : «accès à l'Internet ».

Quant aux avantages de l'utilisation du blog, ils sont beaucoup plus nombreux que les inconvénients, d'après les réponses des participants à l'enquête. Plusieurs étudiants ont mis en évidence la disponibilité du blog et la facilité de l'utiliser : "visible pour tout le monde, accessible de chaque ordinateur et smartphone, facile à utiliser "; " je peux consulter le blog même aujourd'hui, les autres peuvent le consulter, je peux mettre les images et les applications dans un texte, tout le monde peut voir ton blog, tu n'as pas besoin d'USB, c'est plus pratique ». D'autres ont constaté que le blog est "innovatif, dynamique », qu'il incite d'un côté «la motivation, la créativité » et de l'autre côté " la participation à la classe ». Les participants trouvent que cours avec le blog " est plus intéressant qu'un cours ordinaire », que le blog est "ludique, moderne », puis qu'il " motive à travailler » et "donne envie d'apprendre ». L'innovation et la possibilité de varier ses propres pratiques de l'enseignement comptent parmi les plus grands bénéfices apportés par le blog pédagogique. L'innovation et la variation de la pratique apparaissent à la rencontre de la technologie et de l'enseignement. Cette rencontre « développe l'imagination " chez les apprenants et nous fait découvrir "des possibilités illimitées pour créer des activités ».

\subsection{Le blog vu de la part des jeunes concepteurs du blog}

Dans notre pratique de l'enseignement des TICE, nos étudiants, futurs enseignants du FLE, ont été amenés à devenir eux-mêmes les concepteurs des blogs dédiés à l'apprentissage du FLE de différents niveaux (de A1 à B2). D'après leur expérience de concepteurs du blog, on peut constater que les étudiants ont trouvé plus de côtés positifs que négatifs de l'utilisation du blog.

Nos répondants à l'enquête trouvent que les plus grands inconvénients du blog concernent le temps indispensable à sa création ("le temps consacré à l'aspect visuel du blog "; "la création des blogs exige beaucoup de temps 
»), ainsi que son organisation ("l'organisation du blog était un peu difficile »), ce qui est d'ailleurs le défi que chaque enseignant doit surmonter lors de la préparation et de la gestion de chaque cours. D'ailleurs, un étudiant avoue qu' " il était difficile d'organiser le contenu et au début de trouver les parties que nous voulons partager sur le blog parce que c'était la première fois que nous faisions le blog ». L'avis d'un étudiant, concepteur du blog, est que le blog pédagogique a peu d'inconvénients «parce qu'on peut influencer sur toutes les caractéristiques du blog, on peut le construire de la façon qui nous semble la plus appropriée. "

De l'autre côté, les avantages du blog sont multiples. En premier lieu, d'après le constat d'un étudiant il représente " la possibilité d'exprimer la créativité et de s'approcher des étudiants ». Un autre y ajoute : "il permet de créer une histoire, les liens successifs entre les exercices/tâches, les images et liens externes... Il permet d'être un enseignant créatif, de créer des matériaux créatifs ». Il est intéressant de noter qu'un troisième étudiant a trouvé que cette créativité ne concerne pas que l'enseignant, mais aussi l'apprenant : "le blog améliore la créativité chez l'élève ». L'autre avantage du blog est le fait qu'il peut servir d'un moyen d'évaluation et de suivi des progrès des apprenants : " nous pouvons voir toujours le progrès de nos apprenants par exemple ». Ce qui fait la grande distinction entre le blog et les méthodes dites classiques est la disponibilité du blog : "les apprenants peuvent l'utiliser à chaque moment ", c'est-à-dire avant, pendant ou après les cours, puis, en faisant les activités sur le blog et en écrivant directement sur le blog "les élèves peuvent s'organiser à sa propre manière ». Les blogs (et surtout s'il s'agit des blogs de classe) peuvent être très souvent un lieu de collaboration : « il est intéressant de travailler dans un groupe (mais cela dépend de la personne) ». Tout cela va de pair avec «les courants pédagogiques les plus actuels qui privilégient précisément le travail collaboratif, la participation et la construction des connaissances de manière active et dans un contexte réel » (SANCLER 2008 : 141-142).

En somme, les étudiants, qui étaient créateurs de leurs propres blogs dédiés à l'apprentissage du FLE, disent qu'une des caractéristiques les plus positives du blog est qu'on a la possibilité de créer des activités pertinentes pour les apprenants au format texte, vidéo, audio, image directement sur le blog ou en mettant un lien externe qui mène vers une activité créée sur les plateformes spécialisées (Padlet, Kahoot, Nearpod, Educaplay, etc.) et qu'on les publie avec une grande facilité. Certains d'entre eux ont apprécié le fait qu'ils pouvaient créer les activités eux-mêmes «choisir un thème, en fonction des besoins des élèves et de leurs intérêts, puis créer de nombreux jeux intéressants pour les enfants ». Pourtant, les jeunes concepteurs des blogs ont trouvé un problème qui se pose, d'ailleurs, devant presque chaque bloggeur/ 
enseignant, et c'est " le manque de motivation à continuer la création des posts, puisqu'il y a déjà trop de sites similaires ». Un autre obstacle que nous, les enseignants, devons surmonter est le manque de créativité, ou en d'autres termes, le fait qu'il n'y a " pas beaucoup de possibilités pour rendre le texte plus attractif ». Si nous ne sommes pas créatifs et motivés à blogger, il faut donc minimiser cette activité et essayer de trouver dans sa propre boîte à outils d'enseignant d'autres types d'activités en cours avec ou sans les TICE. Il est souhaitable d'utiliser le blog, et les TICE en général, dans la mesure qui convient tant au enseignant qu'aux apprenants.

\section{Conclusion}

Grâce à nos propres pratiques de l'utilisation du blog dans les différents cours nous pouvons constater que cet outil peut être utile et motivant pour les apprenants s'il est bien conçu et s'il rend l'acquisition des compétences linguistiques, pragmatiques et interculturelles plus facile, plus souple et enfin plus innovante. Notre enquête a montré que la grande majorité des apprenants étaient très motivés ou motivés (au total 91,66\%) lorsque nous avons utilisé le blog dans l'enseignement. Le pourcentage total de ceux qui trouvent le blog très utile ou utile à l'apprentissage est encore plus important (au total $95,83 \%$ ). Un des répondants à l'enquête a très bien résumé son expérience en présentant les raisons pour lesquelles le blog est utile : "je le trouve utile, parce que ça brise la monotonie des sujets trop arides. Le concept des cours peut être très intéressant aux étudiants plus âgés, aux petits aussi, je suppose. Je le trouvais innovatif, intéressant, et en même temps éducatif ».

Il existe aussi certains avantages de l'utilisation des blogs pédagogiques pour les enseignants eux-mêmes. La diffusion des contenus pédagogiques sur les blogs a un grand effet positif sur leur pratique d'enseignement : les matériaux rendus publics grâce aux blogs restent à la disposition de tout enseignant en tant que pistes de réflexion et idées à développer. De cette façon l'enseignant crée sa propre boîte à outils qui ne cesse jamais de se développer. En plus, les commentaires des autres enseignants sur le blog peuvent représenter une sorte de feedback sur son travail.

Quant à l'intégration du blog dans les milieux scolaires, il ne s'agit pas seulement de prendre en compte les contraintes techniques et informatiques, il faut repenser les méthodes utilisées et préciser bien les objectifs. Cela est dû au fait que la didactisation des documents authentiques, la création de toutes les activités sur le blog, parfois nécessite certains ajustements, changements, et plus de précisions par rapport aux activités exploitées en cours. Donc, il est vrai que le blog apporte certains changements dans la préparation des cours du 
FLE, qu'il demande des heures supplémentaires de travail, mais la motivation et le progrès des apprenants sont la récompense pour chaque enseignant. Un autre fruit du travail sur le blog est le développement de la créativité chez les apprenants, mais aussi chez l'enseignant, ainsi que l'apparition de meilleurs liens sociaux entre l'enseignant et ses apprenants. Et enfin le temps consacré au blog fait en sorte qu'il y ait un certain prolongement des cours en dehors de l'école, vu que les apprenants font les activités publiées sur le blog à la maison. Les avantages de l'utilisation du blog dans l'enseignement du FLE et dans l'enseignement en général sont multiples. Pourtant, il ne faut pas regarder le blog comme un miracle qui va remédier à tout problème concernant l'acquisition des compétences communicatives. Il n'est pas une méthode à part, mais un outil qui peut nous aider dans notre pratique s'il est utilisé d'une manière appropriée.

\section{Bibliographie}

CAMILLERI, FORD et al. 2007 : CAMILLERI, Mario et Peter FORD, Helena LEJA, Valerie SOLLARS. Blogs dans l'enseignement des langues vivantes. Strasbourg : Éditions du Conseil de l'Europe, 2007.

CAMPBELL 2003 : CAMPBELL, Aaron Patric. « Weblogs for use with ESL classes. » The Internet TESL Journal, Vol. IX, No.2. 2003. < http://iteslj. org/Techniques/Campbell-Weblogs.html>. 05.09.2019.

CORDINA, RAMBERT et al. 2017 : CORDINA, David et Jérôme RAMBERT, Marc ODDOU. Pratiques et projets numériques en classe de FLE. Paris : CLE International, 2017.

DESAVOYE, DUCAMP et al. 2005 : DESAVOYE, Benoît et Christophe DUCAMP, Xavier DE MAZENOD, Xavier MOISANT. Les blogs : nouveau média pour tous. Paris : M2 Editions, 2005.

ENCYCLOPÆDIA UNIVERSALIS [en ligne] 2019 : ENCYCLOPÆDIA UNIVERSALIS [en ligne]. 2019. <http://www.universalis.fr/ encyclopedie/blog/>. 05.08.2019.

HÉNAFF 2009 : HÉNAFF, Nolwenn. « Etude d'un blog pédagogique : Le blog d'une enseignante en histoire-géographie. " Distances et savoirs, vol. 7(3), 377-398. <https://www.cairn.info/revue-distances-et-savoirs2009-3-page-377.htm>. 15.09.2019.

HOECHSMANN, DEWAARD 2015 : HOECHSMANN, Michael et Helen DEWAARD. Définir la politique de littératie numérique et la pratique dans le paysage de l'éducation canadienne. Ottawa : Habilo Médias, 2015.<http://habilomedias.ca/ressources-p\%C3\%A9dagogiques/ utiliser-comprendre-et-cr\%C3\%A9er-un-cadre-de-litt $\% \mathrm{C} 3 \% \mathrm{~A} 9 \mathrm{ratie}-$ num $\%$ C 3\% A 9rique-pour-1es-\%C3\%A9coles-canadiennes/ 
$\mathrm{d} \% \mathrm{C} 3 \%$ A9finir-la-politique-de-litt $\% \mathrm{C} 3 \% \mathrm{~A} 9$ ratie-num $\% \mathrm{C3} \% \mathrm{~A} 9$ riqueet-la-pratique-dans-le-paysage-de-1>. 02.10.2019.

KLEIN (dir.) 2007 : KLEIN, Annabelle. Objectif Blogs ! Explorations dynamiques de la blogosphère. Paris : L'Harmattan, 2007.

LAPOINTE, DROUIN 2007 : DROUIN, Josée Nadia et Pascal LAPOINTE. Science, on blogue !: le nouveau monde d'Internet. Montréal : Les Editions MultiMondes, 2007.

LEBRUN 2002 : LEBRUN, Marcel. Des technologies pour enseigner et apprendre. Bruxelles : De Boeck Université, 2002.

LE MEUR, BEAUVAIS 2005 : LE MEUR, Loïc et Laurence BEAUVAIS. Blogs pour les pros. Paris : Dunod, 2005.

PEDAGOŠKI LEKSIKON 1996 : PEDAGOŠKI LEKSIKON. Beograd: Zavod za udžbenike i nastavna sredstva, 1996. [LEXICON DE PÉDAGOGIE].

LE PETIT ROBERT (Le CD-rom) 2006 : LE PETIT ROBERT (Le CD-rom). Version électronique du Nouveau Petit Robert. Paris : Dictionnaires Le Robert, 2006.

ROBERT 2008 : ROBERT, Jean-Pierre. Dictionnaire pratique de didactique du FLE. Paris : Ophrys, 2008.

ROBOREDO SEARA 2010 : ROBOREDO SEARA, Isabel. « Le weblog : frontières d'un nouveau genre » dans Iliescu M. et al. Actes du XXVe Congrès International de Linguistique et de Philologie Romanes. Walter de Gruyter, 2010.

SANCLER 2008 : SANCLER, Joan Valentina. « Le Wiki, un Outil Web 2.0 dans l'Enseignement du FLE »dans Synergies Venezuela $n^{\circ} 4$. (dir. Y. Quintero de Rincón et V. Bustamante). Maracaibo : GERFLINT, 2008. pp. 140-149.

TRÉDAN 2005 : TRÉDAN, Olivier. Les weblogs dans la Cité : entre quête de l'entre-soi et affirmation identitaire. Môle Armoricain de Recherche sur la Société de l'Information et les Usages d'Internet. <http://www. marsouin.org/IMG/pdf/Tredan_6-2005.pdf>. 12.06.2019.

Стефан Ј. Здравковић

\section{ЕДУКАТИВНИ БЛОГ КАО СРЕДСТВО У НАСТАВИ/УЧЕЮУ ФРАНЦУСКОГ ЈЕЗИКА КАО СТРАНОГ}

У раду анализирамо едукативни блог који се у новој ери технологије појављује као посебно наставно средство, са свим својим предностима и недостацима. Полазећи од дефиниције Матања, Берхина и Орбана 
де Гзиврија (2007) који едукативни блог дефинишу као „праксу и/ или ширење садржаја који потпомажу процесу учења или који из њега произилазе у настави", главни циљ овог рада јесте да потврди или оповргне хипотезу да едукативни блог доноси нове тенденције у припреми часова француског језика као страног, да утиче на повећање мотивације код ученика и да доприноси настанку бољих друштвених веза између аутора блога - наставника и његове публике - ученика̂. У раду испитујемо да ли интеракција између наставника и ученика̂, која настаје у датом новом дигиталном простору обогаћеном мултимедијалним садржајима, ствара нове едукативне потенцијале и одређени продужетак часа. Овај рад се не ограничава само на теоријско одређење овог новог наставног средства, него ће и предложити једну квалитативну анализу едукативних блогова на основу анкете која је спроведена међу студентима који су имали прилике да раде са блогом и као корисници и као аутори сопствених едукативних блогова.

Кључне речи: блог, нове технологије у настави, француски језик као страни, наставник, студент 